\title{
A multi-source towed streamer test in the Pre-Salt
}

Marc Rocke* and Philip Fontana, Polarcus

Copyright 2019, SBGf - Sociedade Brasileira de Geofísica

This paper was prepared for presentation during the $16^{\text {th }}$ International Congress of the Brazilian Geophysical Society held in Rio de Janeiro, Brazil, 19-22 August 2019.

Contents of this paper were reviewed by the Technical Committee of the $16^{\text {th }}$ International Congress of the Brazilian Geophysical Society and do not necessarily represent any position of the SBGf, its officers or members. Electronic reproduction or storage of any part of this paper for commercial purposes without the written consent of the Brazilian Geophysical Society is prohibited.

\section{Abstract}

A 2D test is used to assess the suitability of applying multi-source towed streamer geometries to image deep reflectors below thick, complex salt bodies.

The analysis is focused on the effectiveness of deblending in the presence of salt, and the signal to noise content of multi-source towed streamer data compared to that of a conventional, dual source dataset.

\section{Introduction}

At the core of multi-source technology is the ability to decrease shotpoint interval of sequential shot acquisition by up to a factor of four or five times that of conventional acquisition without the need for additional equipment onboard the vessel. Decreasing shotpoint intervals increases the trace density by an equivalent factor for a given streamer spread, thereby allowing greater flexibility in marine survey design. This has enabled a step change in survey efficiency through the use of wider spreads, and data quality through denser spatial sampling, as well as significantly reducing HSE exposure to the riskiest aspects of towed streamer operations. Two technologies that enable this technique however, deblending in processing and the ability to configure the six gun strings of modern 3D seismic vessels into $3,4,5$, or 6 discreet sources with reduced raw acoustic outputs, have been viewed with skepticism as they relate to salt basins.

Any inherent risks of the deblending process have declined in recent years as the technology matures and is applied on an increasing number of successful commercial projects. However, it has been suggested that any difficulty in distinguishing signal from residual blending noise potentially increases the risk of an inadequate deblending result due to the relatively lower amplitudes returned from pre-salt reflectors as a result of absorption and scattering by salt bodies. Furthermore, the perception that using large volume source arrays as the best way to maximize amplitudes from deep, pre-salt reflectors means that the single vessel, multi-source approach is often overlooked as being too risky for salt.

Nonetheless, a signal to noise approach as the basis for analyzing depth of illumination as opposed to raw source output has been credibly discussed in the past. Musser and Dunbar (1985) first demonstrated that the primary cause of noise in the seismic record is residual shot noise, and that increasing raw source output (often used synonymously with source volume) also increases residual shot noise, without any signal to noise improvement. Langhammer and Bennion (2015) also arrived at a similar conclusion by comparing triple source acquisition to dual source acquisition in the North Sea where both geometries achieved an equivalent signal to noise result based on analysis of signal and noise amplitude spectra calculated on raw stacks.

The objective of the test described in this paper was to analyze and document a deblending result and a signal to noise comparison of dual source versus triple source in a complex salt basin. The benefits of mult-source acquisition to survey efficiency and data quality are not demonstrated, as these are accessed through full 3D application of multi-source geometries, and have been demonstrated in previous publications (Hager et al 2016, and Rocke et al 2017). Instead, we hope to determine whether the multi-source method can be safely applied in the pre-salt, thereby enabling access to the successes achieved in other basins around the world.

\section{Method}

A full production 3D Multi Azimuth (MAZ) survey was acquired over the BMS-50 and Southwest Sagitario blocks in the Santos Basin during the 2016/2017 shooting season. These data were processed to PreSTM onboard the vessel in near real-time with acquisition, as well as to PreSDM as part of the full onshore processing in 2018. The onboard processing was used to select a suitable area for acquisition of a single triple source test line which overlapped a dual source sail-line from the 2016/2017 survey. The area of the test was selected due to the thick and complex nature of the salt as shown in Figure $1 \mathrm{a}$ ). The geometries were configured to achieve two overlapping source lines between the dual source and triple source sail-lines, thereby allowing for a direct comparison of overlapping CMP lines.

The acquisition parameters for the dual source sail-line and the overlapping triple source test line are shown in Table 1 below. For analysis of the test, an equivalent processing sequence were applied to both datasets, including denoise, source and receiver deghosting for broadband, designature and debubble, SRME, highresolution radon demultiple, $2 \mathrm{D}$ PreSDM using the final velocity model from the full onshore processing of the $3 D$ MAZ survey, and post-migration processing. Importantly, deblending was applied to the triple source test line data prior to applying the above processing sequence, but was not required for the dual source dataset. 


\section{Results}

Figure 2 below shows raw, blended common shot gathers from the triple source test line. The water bottom reflection from the primary shot can be seen arriving at around 2.5 seconds on the near offset. With a $12.5 \mathrm{~m}$ shotpoint interval and an average vessel speed of $4.5 \mathrm{kts}$, an average shot period of 5.4 seconds is expected. It should be noted that the time between consecutive water bottom reflections, often referred to as the "unblended zone," is equal to the time between shots, and is thus a function of the shotpoint interval selected during the survey design phase. In the case of this test, the "unblended zone" extends to well below the base of salt and any potential pre-salt reservoirs. This is one approach by which deblending risk has been mitigated in the past and is still applicable should it be necessary after all factors are considered, such as in the case of time lapse targets.

The deblending result on a raw stack can be seen in Figure 3. A characteristic of fixed shotpoint interval, sequential shooting, towed streamer seismic is relatively small variations in the shot period of the acquired data as a result of natural variability in vessel speed in response to waves, currents, and wind. This manifests itself as randomness in the arrival times of overlapping shots when the data is datumed to $T_{0}$ of any one of the multiple sources used, and allows for successful reparation of shots in the deblending process. It is visible on the before deblending stack shown in Figure 3 where the interfering arrivals are seen to vary between 8 seconds and 9 seconds two-way-time. An analysis of the triple source shot periods for the test line revealed a natural localized variability of $+/-400$ milliseconds.

A good indicator of the successful deblending result is apparent on examining the difference plot in Figure 3, where no detectable signal leakage can be seen at the amplitudes of the display. More importantly, the steeply dipping diffraction tails (diffracted multiple in this instance) critical for imaging the faults and steeply dipping salt flanks in the area are perfectly preserved despite having similar moveout to the overlapping energy. In addition, the deep pre-salt reflectors have been revealed by deblending without any detectable signal leakage or residual blending noise at the amplitudes of these plots.

By its very nature, a successful deblending result indicates good signal to noise at depth, in the sense that primary shot energy at depth must be of sufficiently high amplitude relative to background noise to allow the deblending process to successfully distinguish between primary and overlapping shot energy. Further confirmation of the signal to noise characteristics of the triple source test dataset can be found by comparing signal and noise amplitude spectra of the dual source and triple source data on final depth migrated stacks shown in Figure 4. The dual source and triple source data have equivalent signal to noise values of $19 \mathrm{~dB}$ and $29 \mathrm{~dB}$ in the sediment and pre-salt amplitude analysis windows respectively. In the presentation we will show a side-byside comparison of Kirchhoff and RTM PreSDM final stacks for the dual source line and overlapping triple source test line.

\section{Conclusions}

Multi-source towed streamer acquisition achieves significant gains in survey efficiency, data quality, while reducing operational risk. The results of a $2 \mathrm{D}$ test directly comparing triple source and dual source configurations in the Santos Basin Pre-Salt indicates that multi-source data can be successfully deblended and achieves comparable signal to noise characteristics, both for shallow post salt, and deep pre-salt targets. The successful test results indicate that benefits of triple source 3D acquisition can be achieved in the presence of complex salt geology.

\section{Acknowledgments}

We thank Polarcus for permission to share this study and Downunder Geosolutions for deblending and depth migration results.

\section{References}

Ed Hager, Robert Kneale, Laurence Hansen, and Troy Thompson (2016) Baxter: A high-resolution penta-source marine 3D seismic acquisition. SEG Technical Program Expanded Abstracts 2016: pp. 173-177

Ed Hager*, Marc Rocke, and Phil Fontana (2015) Efficient multi-source and multi-streamer configuration for dense cross-line sampling. SEG Technical Program Expanded Abstracts 2015: pp. 100-104

J. Langhammer* and P. Bennion (2015) Triple-Source Simultaneous Shooting (TS3), A Future for Higher Density Seismic? 77th EAGE Conference \& Exhibition

James A. Musser and John A. Dunbar (1984) A quantitative study of source- related noise. SEG Technical Program Expanded Abstracts 1984: pp. 262264

Marc Rocke, Edward Hager, and Philip Fontana (2017) Multisource towed streamer acquisition techniques. SEG Technical Program Expanded Abstracts 2017: pp. 166169 

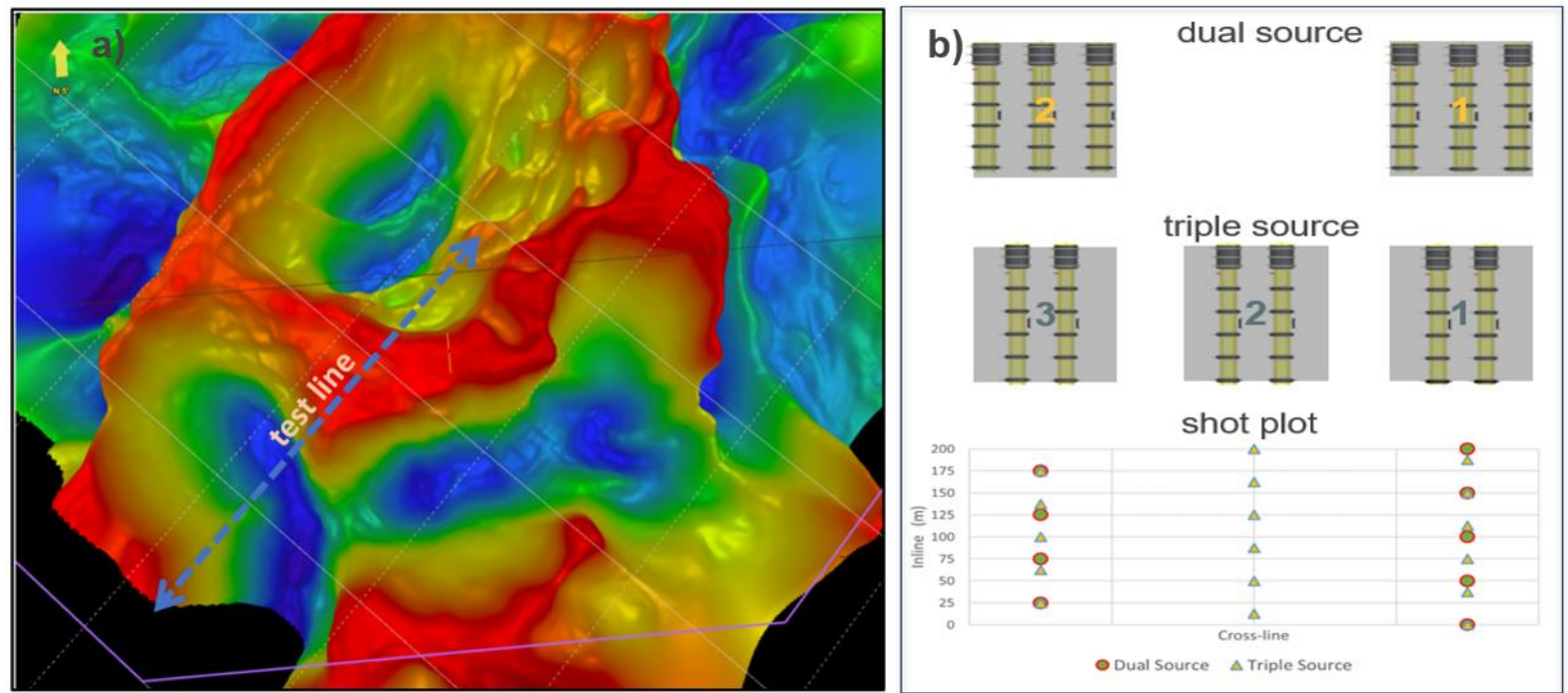

Figure 1: a) Test line location over the Soutwest Sagitario block. b) Illustration of a dual source configuration (above) and triple source configuration (middle) of the vessel's six gun strings, as well as the nominal shot plot of their respective sail lines (below).

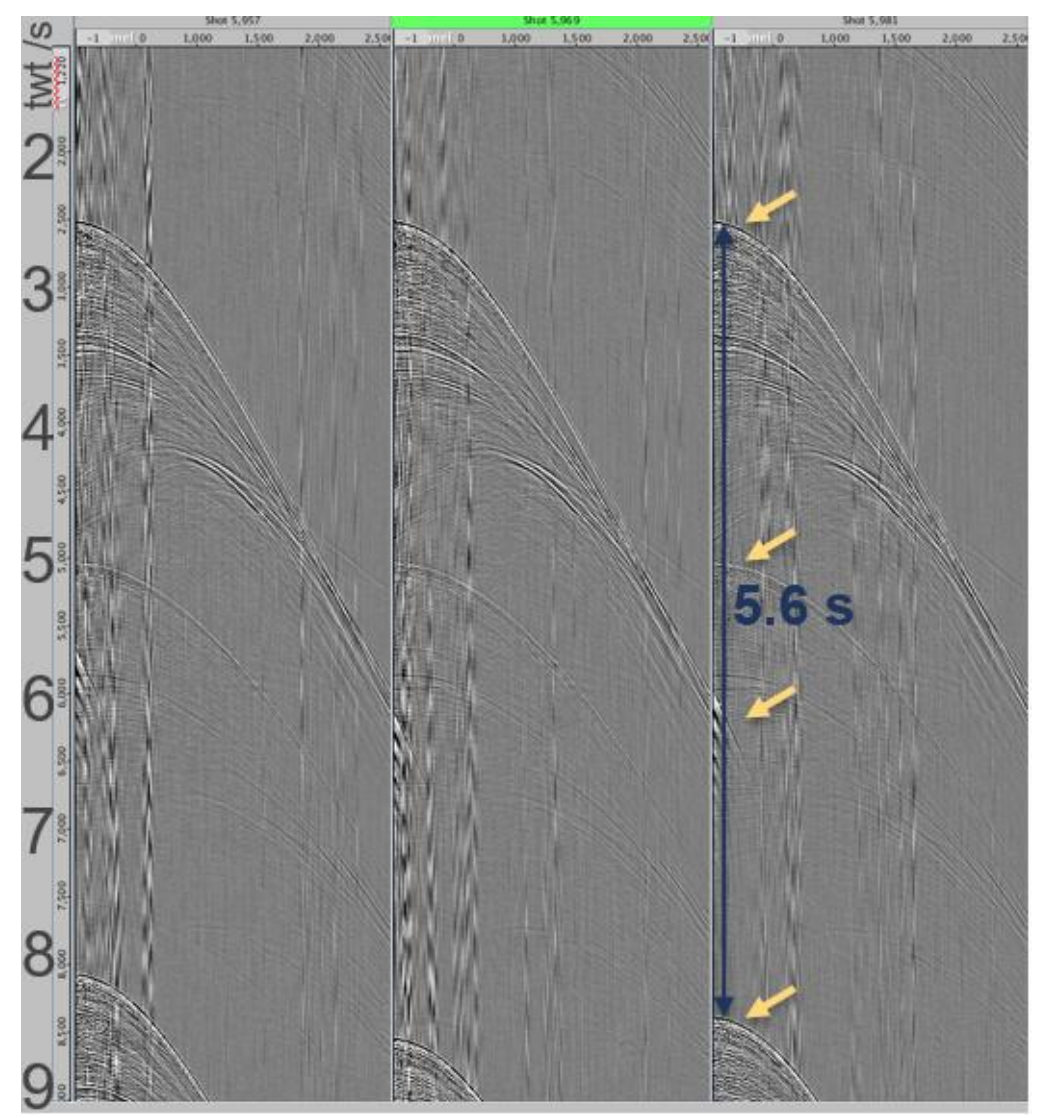

Figure 2: Blended common shot record from the triple source test line showing 5.6 seconds between water bottom reflections of primary and overlapping shots. 


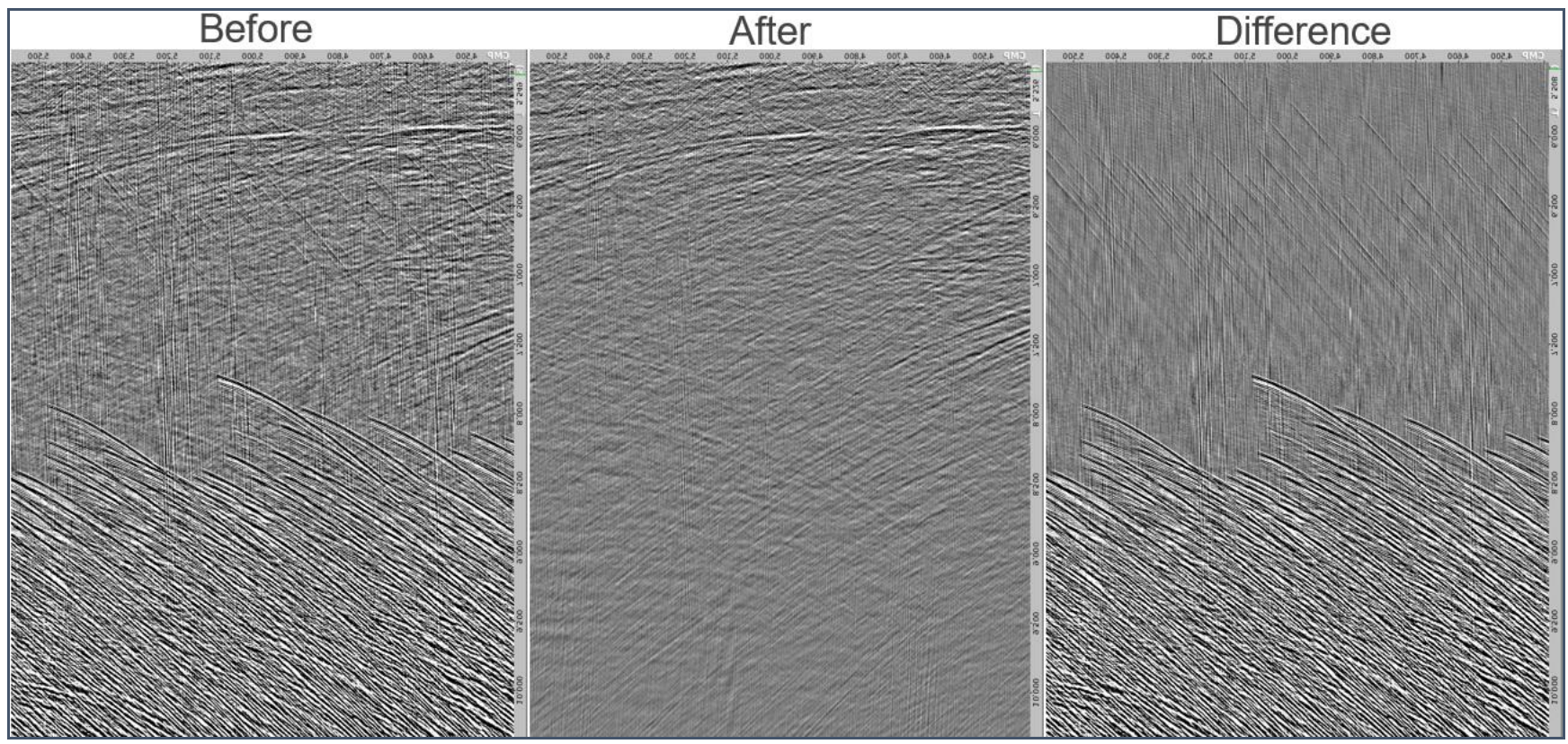

Figure 3: Deblending before, after, and difference stacks of as applied to triple source test line.
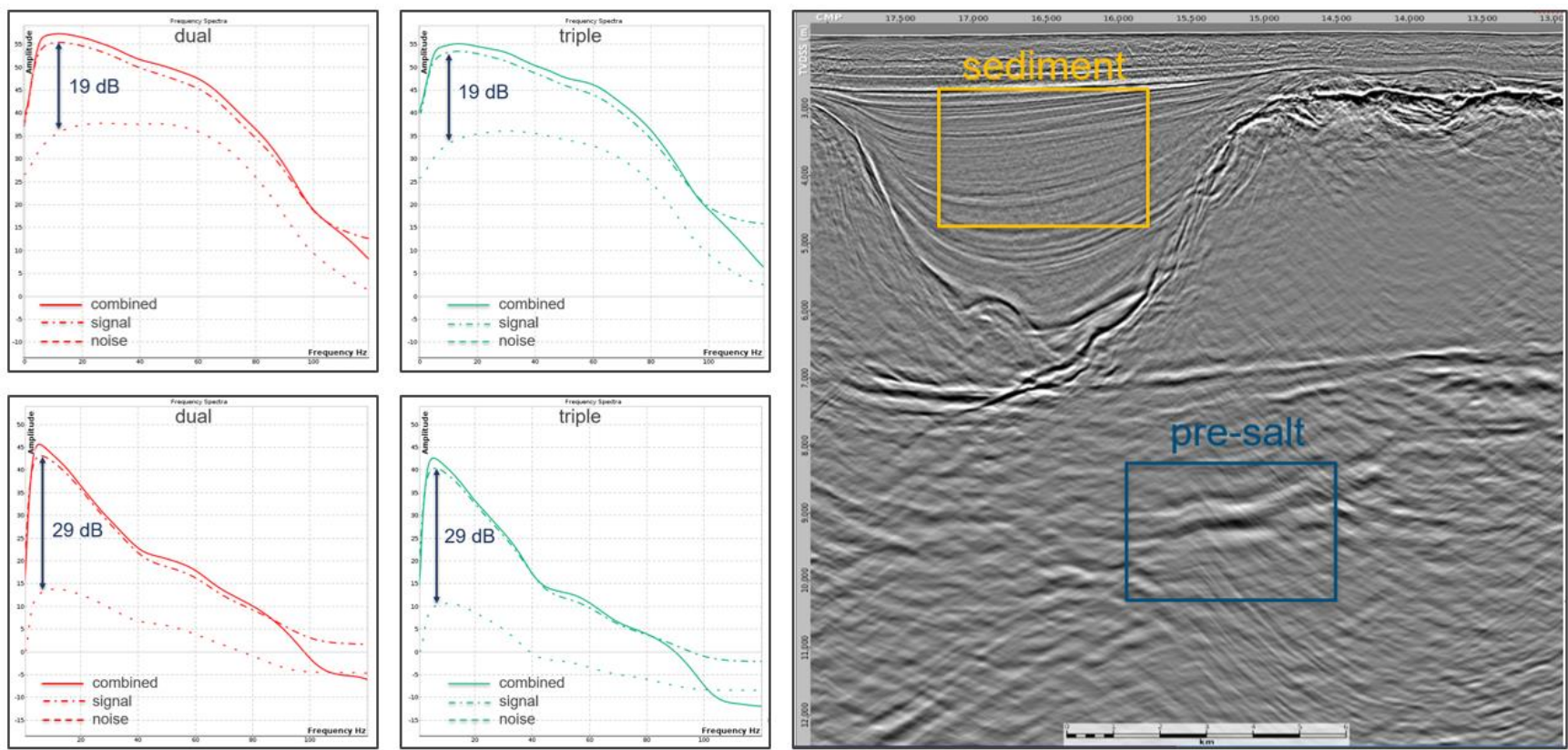

Figure 4: Sediment (top left) and pre-salt (bottom left) signal to noise amplitude spectra extracted from PreSDM stacks of the triple source test line and dual source production line. 


\begin{tabular}{|l|l|l|}
\hline & Dual Source & Triple Source \\
\hline \hline Number of Strings per source & three & two \\
\hline Source Volume (in $\left.{ }^{3}\right)$ & 4240 & 3090 \\
\hline $\begin{array}{l}\text { Source output (bar-m DFSV 0-128 } \\
\text { Hz) }\end{array}$ & $\underline{\mathbf{5 0}}$ & $\underline{\mathbf{3 4}}$ \\
\hline Shot firing mode & Sequential / flip-flop & Sequential / flip-flop-flap \\
\hline Shotpoint interval (m) & $\underline{\mathbf{2 5}}$ & $\underline{\mathbf{1 2 . 5}}$ \\
\hline Shotpoint interval per source (m) & 50 & 37.5 \\
\hline Nominal shot period (s) & $\underline{\mathbf{1 0 . 8}}$ & $\underline{\mathbf{5 . 4}}$ \\
\hline Record Length (s) & 10 & 10 \\
\hline Nominal Fold & 81 & 108 \\
\hline
\end{tabular}

Table 1: Table comparing acquisition parameters of dual source production line and overlapping triple source test line. 